\title{
Rosiglitazone ameliorates acute hepatic injury via activating the Nrf2 signaling pathway and inhibiting activation of the NLRP3 inflammasome
}

\author{
LING MA ${ }^{1}$, YING MA ${ }^{2}$, BIN-XI MA ${ }^{1}$ and MING MA ${ }^{3}$ \\ ${ }^{1}$ Department of Pharmacy, The Eighth Affiliated Hospital of Xinjiang Medical University, Urumqi, Xinjiang 830049; \\ ${ }^{2}$ Department of Clinical Laboratory, The First Affiliated Hospital of Xinjiang Medical University, \\ Urumqi, Xinjiang 830054; ${ }^{3}$ Department of Hepatobiliary Surgery, People's Hospital of \\ Xinjiang Uygur Autonomous Region, Urumqi, Xinjiang 830000, P.R. China
}

Received August 18, 2021; Accepted November 11, 2021

DOI: 10.3892/etm.2022.11229

\begin{abstract}
Acute hepatic injury is a common liver disease in clinical practice. Drugs with antioxidant activity exhibit a great potential for alleviating liver injury. The present study aimed to explore the role of rosiglitazone (RSG), a previously reported compound with anti-inflammatory properties, in hepatic injury. Kunming mice were divided into the following four groups: The control group; the RSG group; the carbon tetrachloride $\left(\mathrm{CCl}_{4}\right)$ group; and the $\mathrm{RSG}+\mathrm{CCl}_{4}$ group. Hepatic injury was confirmed by histological examination of the liver. In addition, the serum levels of alanine transaminase (ALT) and aspartate transaminase (AST), and those of the biochemical indices superoxide dismutase (SOD), catalase (CAT), glutathione (GSH), malondialdehyde (MDA), NO and reactive oxygen species (ROS) were measured in each group of mice. Additionally, the levels of inflammatory factors and apoptosis-related proteins, as well as the activity of the related signaling pathways, were evaluated. The results showed that RSG could reverse the $\mathrm{CCl}_{4}$-mediated decrease in the levels of SOD, CAT and GSH, and increase in the levels of ALT, AST, MDA, NO and ROS. Furthermore, treatment with RSG could reduce the expression levels of inflammation- and apoptosis-related proteins, thus suggesting that RSG could attenuate inflammation and liver cell apoptosis. Additionally, treatment with RSG promoted the activation of the nuclear factor erythroid 2-related factor 2 (Nrf2) signaling pathway, upregulated peroxisome proliferator-activated receptor $\gamma$ and inhibited activation of the inflammasome NOD-like receptor
\end{abstract}

Correspondence to: Dr Ming Ma, Department of Hepatobiliary Surgery, People's Hospital of Xinjiang Uygur Autonomous Region, 91 Tianchi Road, Urumqi, Xinjiang 830000, P.R. China E-mail: maming814@163.com

Key words: rosiglitazone, acute hepatic injury, nuclear factor erythroid 2-related factor 2, NOD-like receptor protein 3, carbon tetrachloride protein 3 (NLRP3). In conclusion, the current study demonstrated that RSG could ameliorate acute hepatic injury via activating the Nrf2 signaling pathway and inhibiting activation of the NLRP3 inflammasome. The findings of the present study partly uncovered the mechanism underlying the effect of RSG on hepatic injury, thus supporting the application of RSG in clinical practice.

\section{Introduction}

Acute hepatic injury is a common liver disease that may lead to hepatic failure. This life-threatening condition may irreversibly affect the normal liver function (1). Several harmful substances can cause acute hepatic injury, including carbon tetrachloride $\left(\mathrm{CCl}_{4}\right)$, alcohol and acetaminophen-based medicines (2). $\mathrm{CCl}_{4}$ is a chemical hepatic toxin that induces liver cell damage via the metabolic activation of cytochrome $\mathrm{P} 4502 \mathrm{E} 1$ (3). In addition, $\mathrm{CCl}_{4}$ triggers metabolism to form free radicals, which in turn can promote the generation of lipid peroxidation products, thus promoting hepatocyte apoptosis and necrosis (4). Therefore, it has been reported that chemicals or drugs with antioxidant activities may possess significant potential for alleviating $\mathrm{CCl}_{4}$-induced hepatic damage (5). The current therapeutic approaches for treating liver diseases primarily focus on the immediate discontinuation of drugs that affect the nature of hepatic damage and the administration of agents with anti-inflammatory properties, acting against free radicals, and those that promote liver cell metabolism, repair and regeneration (6). However, specific and effective treatment strategies against liver diseases are still lacking. Due to its poor prognosis and high mortality, hepatic injury has become a focus in international research (7).

Rosiglitazone (RSG), an insulin sensitizer, has been used to treat type 2 diabetes in clinical practice (8). It can increase the insulin sensitivity of tissues such as skeletal muscle and liver, and improve the utilization of glucose (9). In addition, RSG is a highly selective synthetic ligand of peroxisome proliferator-activated receptor $\gamma$ (PPAR $\gamma$ ); binding of RSG with PPAR $\gamma$ has been shown to upregulate the expression of PPAR $\gamma$ to exert its biological effects (10). A previous study on 
animal models of status epilepticus showed that treatment with RSG could activate PPAR $\gamma$, which in turn may significantly inhibit the production of reactive oxygen species (ROS) and lipid peroxidation, while increasing the antioxidant effects of superoxide dismutase (SOD) and glutathione (GSH) (11). In addition, pretreatment of thrombin-induced microglial cells with RSG has been reported to increase the expression of PPAR $\gamma$ and heme oxygenase 1 (HO-1), thereby increasing their antioxidant ability (12). Furthermore, another study demonstrated that RSG could upregulate nuclear factor erythroid 2-related factor 2 (Nrf2) and heme oxygenase 1 (HO-1) in a PPAR $\gamma$-dependent manner, eliminate excess ROS production and attenuate the oxidative stress response of glucose-induced liver cells (13). The aforementioned studies indicated that PPAR $\gamma$ and its agonist RSG could serve a significant role in regulating the redox state both in vitro and in vivo.

The use of $\mathrm{CCl}_{4}$ as an inducer for establishing a hepatic injury research model has been widely applied and certified (14). Therefore, in the present study, $\mathrm{CCl}_{4}$ was used to induce acute hepatic injury in mice. Subsequently, the effects of RSG on hepatic injury and its underlying mechanism of action were explored. The findings of the current study could provide a novel direction for the treatment of hepatic injury.

\section{Materials and methods}

Animals and reagents. All animal experiments were performed in accordance with the National Institutes of Health Guidelines for the Handling of Laboratory Animals (15) and were approved by the Ethics Committee of The Eighth Affiliated Hospital of Xinjiang Medical University (Urumqi, China; approval no. 2020-035). A total of 40 male Kunming (KM) mice (weight, $20 \pm 2$ g; 4 weeks-old; Beijing Vital River Laboratory Animal Technology Co., Ltd.) were acclimated for 1 week in an environment of $25^{\circ} \mathrm{C}$ with a 12-h light/dark cycle, relative humidity of 55\%, and free access to food and water. Subsequently, $1 \mathrm{ml} \mathrm{CCl}_{4}$ (Sinopharm Chemical Reagent Co., Ltd.) was dissolved in $100 \mathrm{ml}$ olive oil and mixed well to prepare a $1 \% \mathrm{CCl}_{4}$ solution. RSG was purchased from Chengdu Hengrui Pharmaceutical Co., Ltd.

Establishment of the mouse model. KM mice were divided into the following four groups: i) Control; ii) $\mathrm{RSG}$; iii) $\mathrm{CCl}_{4}$; and iv) $\mathrm{RSG}+\mathrm{CCl}_{4}$ groups. Mice in the control and $\mathrm{CCl}_{4}$ group were separately treated with an intraperitoneal injection of a single dose of saline or $1 \% \mathrm{CCl}_{4}(10 \mathrm{ml} / \mathrm{kg})$, respectively. Prior to $\mathrm{CCl}_{4}$ injection, mice in the $\mathrm{RSG}+\mathrm{CCl}_{4}$ group were treated with $10 \mathrm{mg} / \mathrm{kg} \mathrm{RSG}$ by oral gavage for 5 consecutive days. Mice in the RSG group were treated as outlined in the $\mathrm{RSG}+\mathrm{CCl}_{4}$ group, except with an additional $(10 \mathrm{ml} / \mathrm{kg})$ saline injection in place of $\mathrm{CCl}_{4}$. All mice were fasted and had only free access to water for $24 \mathrm{~h}$ after modeling. Subsequently, mice were anesthetized using sodium pentobarbital $(100 \mathrm{mg} / \mathrm{kg}$; intraperitoneal) and the abdominal cavity was opened through an incision along the midline of the abdomen to expose the abdominal aorta. Following blood collection $(0.5 \mathrm{ml})$ with a syringe, the mice were sacrificed by cervical dislocation. The liver tissues were immediately obtained, washed with PBS and were then stored at $-80^{\circ} \mathrm{C}$.
Hematoxylin and eosin $(H \& E)$ staining. The liver tissue samples were fixed in $4 \%$ paraformaldehyde at $4^{\circ} \mathrm{C}$ for $6 \mathrm{~h}$, dehydrated with gradient alcohol and embedded in paraffin. Subsequently, the tissue samples were cut into 5- $\mu \mathrm{m}$ slices. Sections were immersed in xylene, rehydrated with gradient alcohol and water, and stained with hematoxylin for $3 \mathrm{~min}$ and eosin for $3 \mathrm{~min}$ (Beijing Solarbio Science \& Technology Co., Ltd.) at room temperature. Following dehydration with gradient alcohol, the tissues were treated with xylene for $3 \mathrm{~min}$. Physiological changes in the tissue samples were observed under a light microscope (magnification, $\mathrm{x} 400$; Olympus Corporation).

Detection of alanine aminotransferase (ALT) and aspartate aminotransferase (AST). The blood was allowed to stand at $4^{\circ} \mathrm{C}$ for $30 \mathrm{~min}$ and the serum was collected following centrifugation at $1,000 \mathrm{x}$ g for $10 \mathrm{~min}$ at $4^{\circ} \mathrm{C}$. The serum levels of ALT and AST were measured using ALT (cat. no. C009-1-1) and AST assay kits (cat. no. C010-1-1; both from Nanjing Jiancheng Bioengineering Institute), according to the manufacturer's instructions. Briefly, the serum sample was added to a tube, mixed with ALT or AST matrix solution for $30 \mathrm{~min}$ in a $37^{\circ} \mathrm{C}$ water bath followed by the addition of a color developing agent. The reaction was terminated following incubation of the samples with stop solution for $20 \mathrm{~min}$ in a water bath at $37^{\circ} \mathrm{C}$. After incubation for $5 \mathrm{~min}$ at room temperature, a microplate reader (Thermo Fisher Scientific, Inc.) was used to measure the optical density (OD) value of each well at a wavelength of $505 \mathrm{~nm}$.

Detection of indices of hepatic function. The mass of tissue samples used to measure indices of hepatic function was calculated according to the formula: Liver mass (g): volume $(\mathrm{ml})=1: 9$. Subsequently, the tissue samples were added into pre-cooled physiological saline and homogenized on ice. The homogenate was then centrifuged at $1,500 \mathrm{xg}$ at $4^{\circ} \mathrm{C}$ for $10 \mathrm{~min}$ and the supernatant was collected. SOD (cat. no. A001-1), catalase (CAT; cat. no. A007-1-1), GSH (cat. no. A005-1), NO (cat. no. A012-1-2) and malondialdehyde (MDA; cat. no. A003-1-1) assay kits (all from Nanjing Jiancheng Bioengineering Institute) were used to assess the content of the corresponding indices, according to the manufacturer's instructions. The OD values were measured using a microplate reader (Thermo Fisher Scientific, Inc.) at a wavelength of 550 (for SOD and NO), 405 (for CAT), 412 (for GSH) and $532 \mathrm{~nm}$ (for MDA).

The determination of ROS was performed using a ROS assay kit (cat. no. HR7814; Beijing BioRab Technology Co. Ltd.). The tissue sections were soaked in the cleaning solution for $15 \mathrm{sec}$ and then the residual solution on the surfaces were wiped off. The sections were incubated in the staining working solution for $30 \mathrm{~min}$ at $37^{\circ} \mathrm{C}$ in the dark. After washing twice with PBS, the results were observed under a fluorescence microscope (magnification, x100; Olympus Corporation).

ELISA. Serum samples were collected as aforementioned. The secretion levels of TNF- $\alpha$ (cat. no. 88-7324), IL-6 (cat. no. 88-7064) and IL-1 $\beta$ (cat. no. 88-7013; all from Thermo Fisher Scientific, Inc.) in the serum were determined using the corresponding ELISA kits according to the manufacturer's 
Table I. Primer sequences.

\begin{tabular}{ll}
\hline Gene & \multicolumn{1}{c}{ Sequence } \\
TNF- $\alpha$ & F: 5'-GCTGAGCTCAAACCCTGGTA-3' \\
& R: 5'-CGGACTCCGCAAAGTCTAAG-3' \\
IL-6 & F: 5'-CCAGTTGCCTTCTTGGGACTG-3' \\
& R: 5'-CAGGTCTGTTGGGAGTGGTATCC-3' \\
IL-1 $\beta$ & F: 5'-TGCCACCTTTTGACAGTGATG-3' \\
& R: 5'-AGTCACAGAGGATGGGCTCT-3' \\
Nrf2 & F: 5'-ACAGTGCTCCTATGCGTGAA-3' \\
& R: 5'-GAGCCTCTAAGCGGCTTGAA-3' \\
NQO1 & F: 5'-GTCCATTCCAGCTGACAACCA-3' \\
& R: 5'-TTGCCCTGAGGCTCCTAATC-3' \\
HO-1 & F: 5'-TGCTAGCCTGGTGCAAGATA-3' \\
& R: 5'-GCCAACAGGAAGCTGAGAGT-3' \\
GAPDH & F: 5'-TGTTTCCTCGTCCCGTAGA-3' \\
& R: 5'-ATCTCCACTTTGCCACTGC-3'
\end{tabular}

F, forward; R, reverse; Nrf2, nuclear factor erythroid 2-related factor 2; NQO1, NAD(P)H quinone oxidoreductase 1; HO-1, heme oxygenase 1 .

instructions. Finally, a microplate reader (Thermo Fisher Scientific, Inc.) was used to measure the OD values in each well at a wavelength of $450 \mathrm{~nm}$.

Reverse transcription-quantitative PCR (RT-qPCR). Total RNA was extracted from tissues using TRIzol ${ }^{\circledR}$ reagent (Invitrogen; Thermo Fisher Scientific, Inc.). The extracted RNA was reverse transcribed into cDNA using the PrimeScript RT reagent kit (Takara Bio, Inc.) according to the manufacturer's protocol. The mRNA expression levels were quantified using the QuantiTect SYBR Green PCR kit (Qiagen, Inc.) on the ABI 7500 system (Applied Biosystems; Thermo Fisher Scientific, Inc.). The qPCR amplification conditions were as follows: $95^{\circ} \mathrm{C}$ for $10 \mathrm{~min}$, followed by 40 cycles at $95^{\circ} \mathrm{C}$ for $30 \mathrm{sec}, 64^{\circ} \mathrm{C}$ for $34 \mathrm{sec}$ and $72^{\circ} \mathrm{C}$ for $30 \mathrm{sec}$. The relative gene expression levels were normalized to that of the housekeeping gene, GAPDH. The $2^{-\Delta \Delta \mathrm{Cq}}$ method was utilized (16). The primer sequences used for qPCR are listed in Table I.

Western blot analysis. Total proteins were extracted from liver tissue samples using RIPA lysis buffer (Beyotime Institute of Biotechnology). The protein concentration was measured using a BCA protein assay kit (cat. no. A045-3-2; Nanjing Jiancheng Bioengineering Institute). Total protein extracts (20 $\mu \mathrm{g} /$ lane) were then separated by SDS-PAGE on $10 \%$ gels and were transferred onto PVDF membranes. After blocking with $5 \%$ skimmed milk at room temperature for $2 \mathrm{~h}$, the membranes were incubated with primary antibodies against cleaved caspase- 8 (cat. no. 9748; dilution, 1:1,000), caspase-8 (cat. no. 4927; dilution, 1:1,000; both from Cell Signaling Technology, Inc.), cleaved caspase-3 (cat. no. ab32042; dilution, 1:1,000), caspase-3 (cat. no. ab184787; dilution, $1: 2,000)$, cleaved poly(ADP-ribose) polymerase (PARP; cat. no. ab32064; dilution, 1:1,000), PARP (cat. no. ab191217; dilution, 1:1,000), PPAR $\gamma$ (cat. no. ab178860; dilution, 1:1,000), Nrf2 (cat. no. ab62352; dilution, 1:1,000), NAD(P)H quinone oxidoreductase 1 (NQO1; cat. no. ab213239; dilution, 1:500), HO-1 (cat. no. ab52947; dilution, 1:2,000), NOD-like receptor protein 3 (NLRP3; cat. no. ab263899; dilution, 1:1,000), caspase-1 (cat. no. ab138483; dilution, 1:1,000), IL-1 $\beta$ (cat. no. ab234437; dilution, 1:1,000), apoptosis-associated speck-like protein (ASC; cat. no. ab283684; dilution, 1:1,000) and the loading control, $\beta$-actin (cat. no. ab8226; dilution, 1:1,000; all from Abcam) at $4^{\circ} \mathrm{C}$ overnight. Following incubation with the primary antibodies, the membranes were incubated with HRP-conjugated anti-rabbit (cat. no. ab97051; dilution, 1:10,000) or anti-mouse (cat. no. ab6728; dilution, 1:2,000; both from Abcam) antibodies at $37^{\circ} \mathrm{C}$ for $2 \mathrm{~h}$. The bands were visualized with an ECL reagent (MilliporeSigma) at room temperature for $2 \mathrm{~min}$. The density of each band was semi-quantified using ImageJ software (v1.8; National Institutes of Health).

Statistical analysis. All experiments were repeated three times. Data are expressed as the mean \pm SD. One-way ANOVA followed by Tukey's post hoc test was carried out to compare the differences among multiple groups. Statistical analysis was performed using GraphPad Prism 8.0 software (GraphPad Software, Inc.). $\mathrm{P}<0.05$ was considered to indicate a statistically significant difference.

\section{Results}

RSG attenuates hepatic injury. The changes in liver histology among the four groups of mice were evaluated by $H \& E$ staining. The results showed that hepatocytes in the control and RSG groups were a normal shape and were tightly arranged. In the $\mathrm{CCl}_{4}$ group, the cell arrangement was disordered, and inflammatory cell infiltration and cell necrosis were observed. However, pretreatment of $\mathrm{CCl}_{4}$-treated mice with RSG markedly improved inflammatory infiltration. In addition, the cell arrangement was more compact in the $\mathrm{RSG}+\mathrm{CCl}_{4}$ group compared with that in the $\mathrm{CCl}_{4}$ group (Fig. 1A). Furthermore, no differences were observed in the serum levels of ALT and AST between the control and RSG groups. However, in the $\mathrm{CCl}_{4}$ group, the levels of both transaminases were significantly increased, whereas the levels were reduced following pretreatment with RSG (Fig. 1B). Subsequently, the levels of the hepatic biochemical indicators SOD, CAT, GSH, MDA, NO and ROS were determined in each group of mice using the corresponding kits. No statistically significant differences in the activity of the aforementioned indicators were observed between the control and RSG groups. However, the levels of SOD, CAT and GSH were reduced, whereas those of MDA and $\mathrm{NO}$ were increased in the $\mathrm{CCl}_{4}$ group. This effect was abrogated following pretreatment with RSG (Fig. 1C). In addition, the ROS fluorescence of the $\mathrm{CCl}_{4}$ group was significantly enhanced compared with that in the control group and RSG pretreatment reduced the ROS fluorescence intensity (Fig. 1D). These findings suggested that pretreatment of mice with RSG could reduce the severity of $\mathrm{CCl}_{4}$-induced hepatic injury. 
A

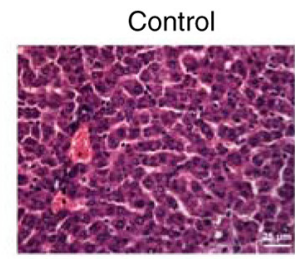

$\mathrm{CCl} 4$

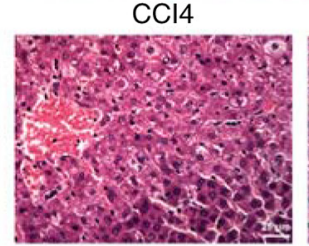

RSG

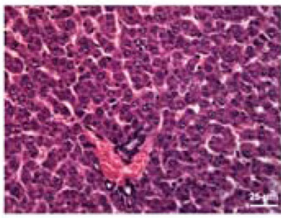

$\mathrm{RSG}+\mathrm{CCl} 4$

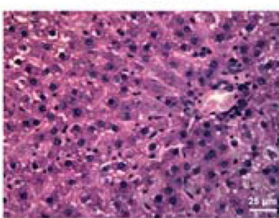

$\mathrm{B}$

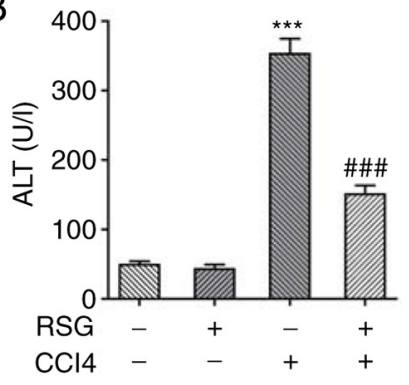

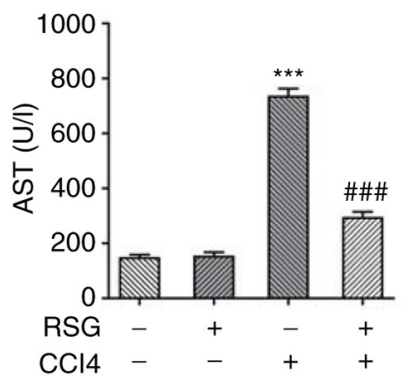

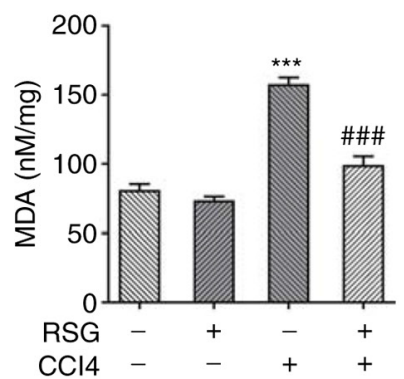

$\mathrm{CCl} 4$
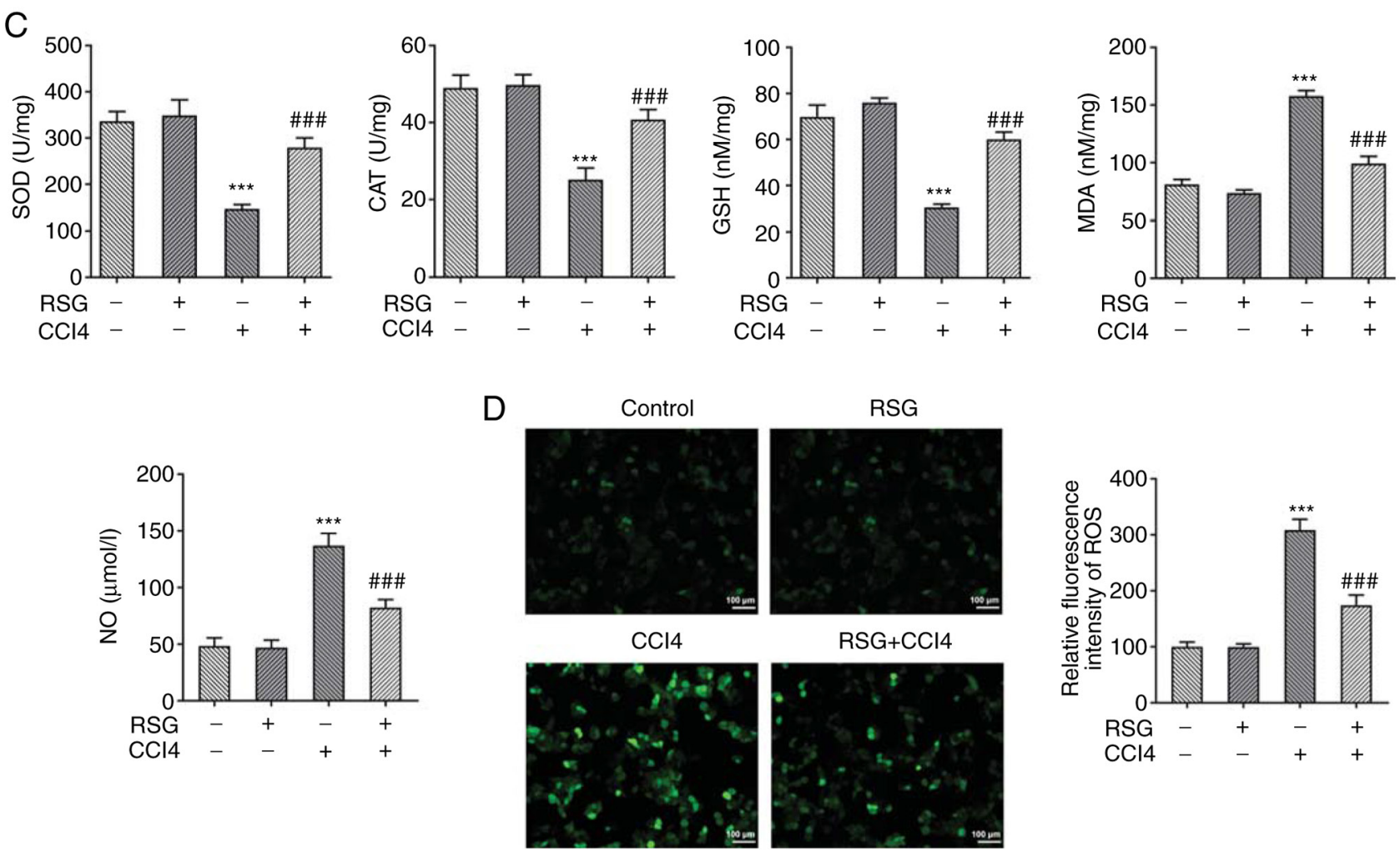

Figure 1. (A) Hepatic tissue samples from the four groups of mice were evaluated by hematoxylin and eosin staining (magnification, $\mathrm{x} 400$ ). (B) ALT and AST levels in the serum samples from each group of mice were determined using the corresponding kits. (C) Hepatic biochemical indicator levels (SOD, CAT, GSH, MDA and NO) in each group of mice were measured using the corresponding kits. (D) ROS levels in each group of mice were measured using an ROS assay kit. ${ }^{* * *} \mathrm{P}<0.001$ vs. the control group; ${ }^{\# \#} \mathrm{P}<0.001$ vs. the $\mathrm{CCl}_{4}$ group. RSG, rosiglitazone; $\mathrm{CCl}_{4}$, carbon tetrachloride; ALT, alanine transaminase; AST, aspartate transaminase; SOD, superoxide dismutase; CAT, catalase; GSH, glutathione; MDA, malondialdehyde; ROS, reactive oxygen species.

RSG may abrogate the effects of $\mathrm{CCl}_{4}$ on hepatocyte inflammation and apoptosis. Since hepatic injury is closely associated with inflammation, the present study aimed to determine the expression levels of inflammatory factors in serum samples and liver tissues isolated from mice in different groups. Therefore, the secretion levels of TNF- $\alpha$, IL- 6 and IL- $1 \beta$ in the serum were measured using ELISA. The secretion levels of the aforementioned inflammatory factors were all enhanced in the $\mathrm{CCl}_{4}$ group compared with those in the control group. However, the levels of TNF- $\alpha$, IL-6 and IL-1 $\beta$ were reduced in the $\mathrm{RSG}+\mathrm{CCl}_{4}$ group compared with the $\mathrm{CCl}_{4}$ group (Fig. 2A). The mRNA expression levels of TNF- $\alpha$, IL- 6 and IL- $1 \beta$ in the liver tissues were detected using RT-qPCR. No statistically significant differences were observed in the expression levels of the inflammatory factors between the control and RSG groups, whereas the increase in the expression of these factors was less prominent in the $\mathrm{RSG}+\mathrm{CCl}_{4}$ group compared with the $\mathrm{CCl}_{4}$ group (Fig. 2B). Additionally, the expression levels of apoptosis-related proteins were evaluated by western blot analysis. Cleaved capase- 3 , cleaved caspase- 8 and cleaved PARP were all upregulated in the $\mathrm{CCl}_{4}$ group compared with in the control group. In addition, the expression levels of the apoptosis-related proteins were reduced in the $\mathrm{RSG}+\mathrm{CCl}_{4}$ group compared with those in the $\mathrm{CCl}_{4}$ group, thus suggesting the RSG may protect hepatocytes from apoptosis to a certain degree (Fig. 2C).

RSG exerts its effects on hepatocyte injury via the Nrf2 and NLRP3 signaling pathways. The aforementioned results 
A

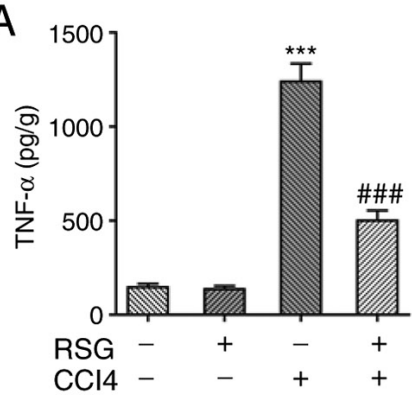

B

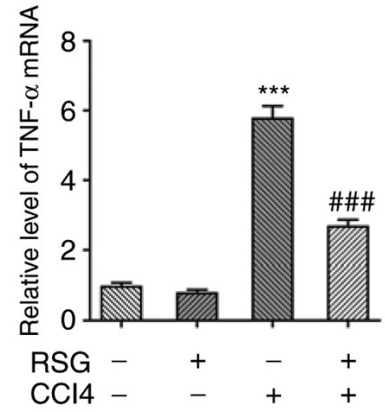

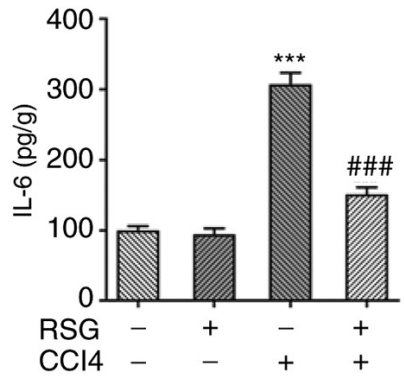

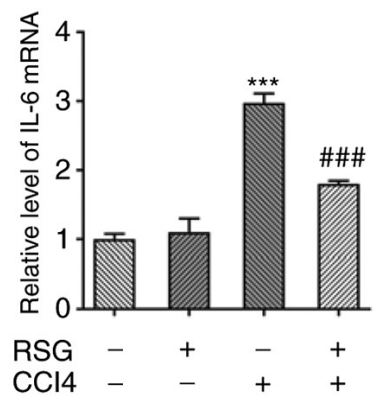

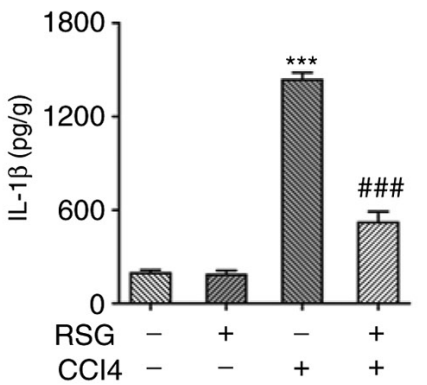

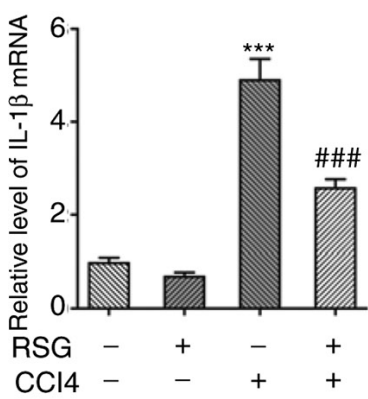

C

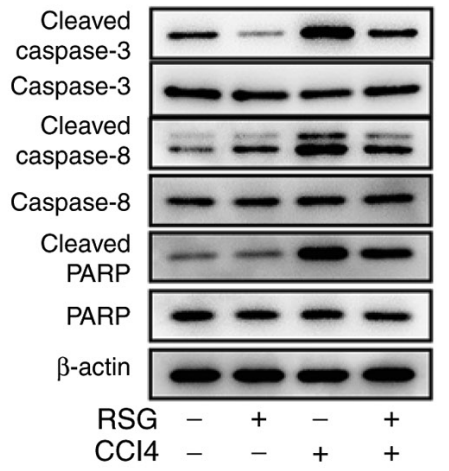

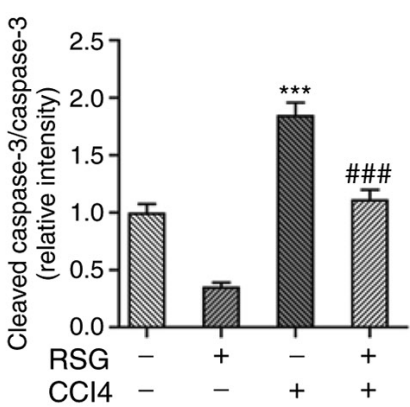

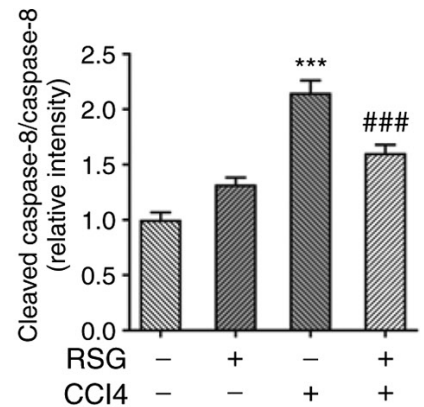

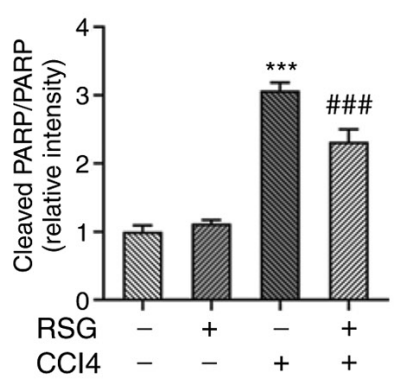

Figure 2. (A) Serum levels of TNF- $\alpha$, IL-6 and IL-1 $\beta$ were determined using the corresponding ELISA kits. (B) mRNA expression levels of TNF- $\alpha$, IL- 6 and IL-1 $\beta$ in tissue samples were determined using reverse transcription-quantitative PCR. (C) Expression levels of the apoptosis-related proteins were measured using western blot analysis. ${ }^{* * *} \mathrm{P}<0.001$ vs. the control group; ${ }^{\# \#} \mathrm{P}<0.001$ vs. the $\mathrm{CCl}_{4}$ group. $\mathrm{RSG}$, rosiglitazone; $\mathrm{CCl}$, carbon tetrachloride; $\mathrm{PARP}$, poly(ADP-ribose) polymerase.

indicated that RSG could alleviate hepatic injury; therefore, subsequent experiments focused on uncovering the possible mechanism underlying the effects of RSG on hepatic injury. The expression levels of PPAR $\gamma$ and of proteins associated with the Nrf2 signaling pathway were determined using western blot analysis. The results showed that PPAR $\gamma, \mathrm{Nrf2}, \mathrm{NQO} 1$ and $\mathrm{HO}-1$ were downregulated in the $\mathrm{CCl}_{4}$ group compared with in the control group. However, pretreatment with RSG reversed the $\mathrm{CCl}_{4}$-mediated decreased expression of these factors (Fig. 3A). The mRNA expression levels of Nrf2, NQO1 and HO-1 were measured by RT-qPCR. Consistently, the expression levels of $\mathrm{Nrf} 2$, NQO1 and HO-1 were downregulated in the $\mathrm{CCl}_{4}$ group and were restored following pretreatment with RSG (Fig. 3B). Additionally, the expression levels of the NLRP3 signaling pathway-related proteins were detected using western blot analysis. NLRP3, caspase-1, IL-1 $\beta$ and ASC were significantly upregulated in the $\mathrm{CCl}_{4}$ group compared with those in the control group. However, the expression these factors was significantly decreased in the $\mathrm{RSG}+\mathrm{CCl}_{4}$ group compared with in the $\mathrm{CCl}_{4}$ group (Fig. 4).

\section{Discussion}

Currently, inhibiting oxidative stress and inflammation is considered a significant strategy for treating hepatic injury (17). It has been reported that the $\mathrm{CCl}_{4}$-induced chemical hepatic injury model mimics the pathology of human viral hepatic injury (18). Therefore, $\mathrm{CCl}_{4}$ was selected to establish a mouse model of hepatic injury. In the present study, following histopathological examination, the serum levels of ALT and AST were measured. ALT and AST are two enzymes that play a significant role in amino acid metabolism and are widely present in hepatocytes. The inflammation-, necrosis- or poisoning-mediated hepatocyte injury can change the permeability of hepatic cellular membrane, thus promoting the overflow of ALT and AST from the cells into the blood circulation (19). In the present study, the serum levels of ALT and AST were significantly increased in $\mathrm{CCl}_{4}$-treated mice, whereas pretreatment with RSG abrogated this effect. Furthermore, the levels of several hepatic indicators were determined, including SOD, CAT, GSH, MDA, NO and ROS. The activity of SOD, an active protease containing 
A

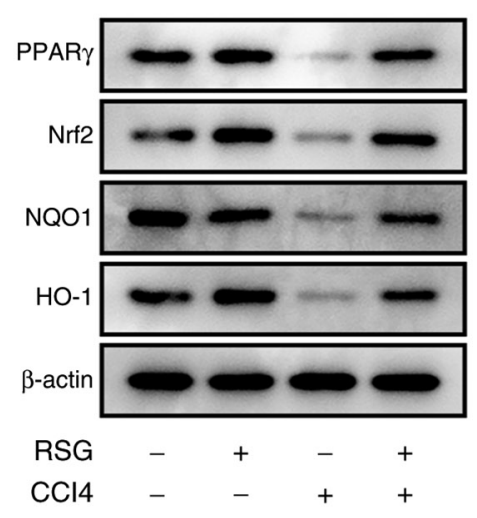

B

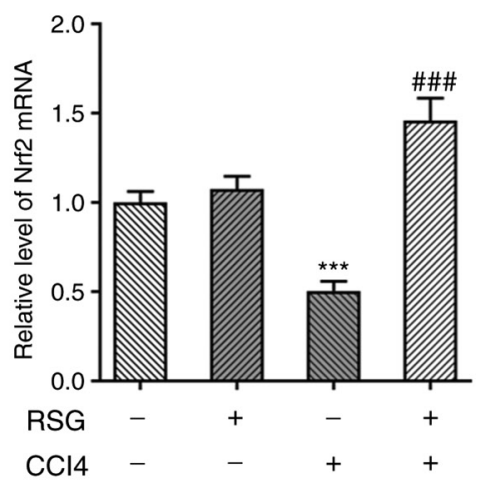

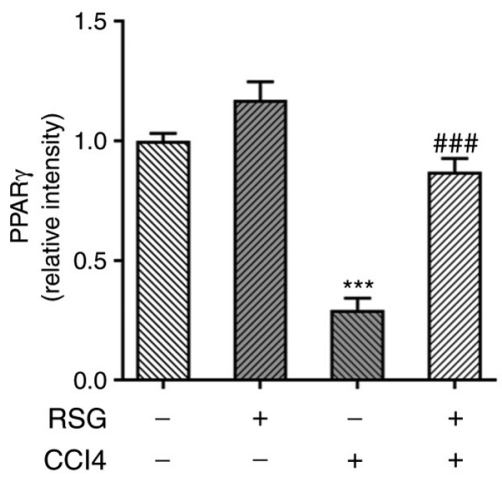
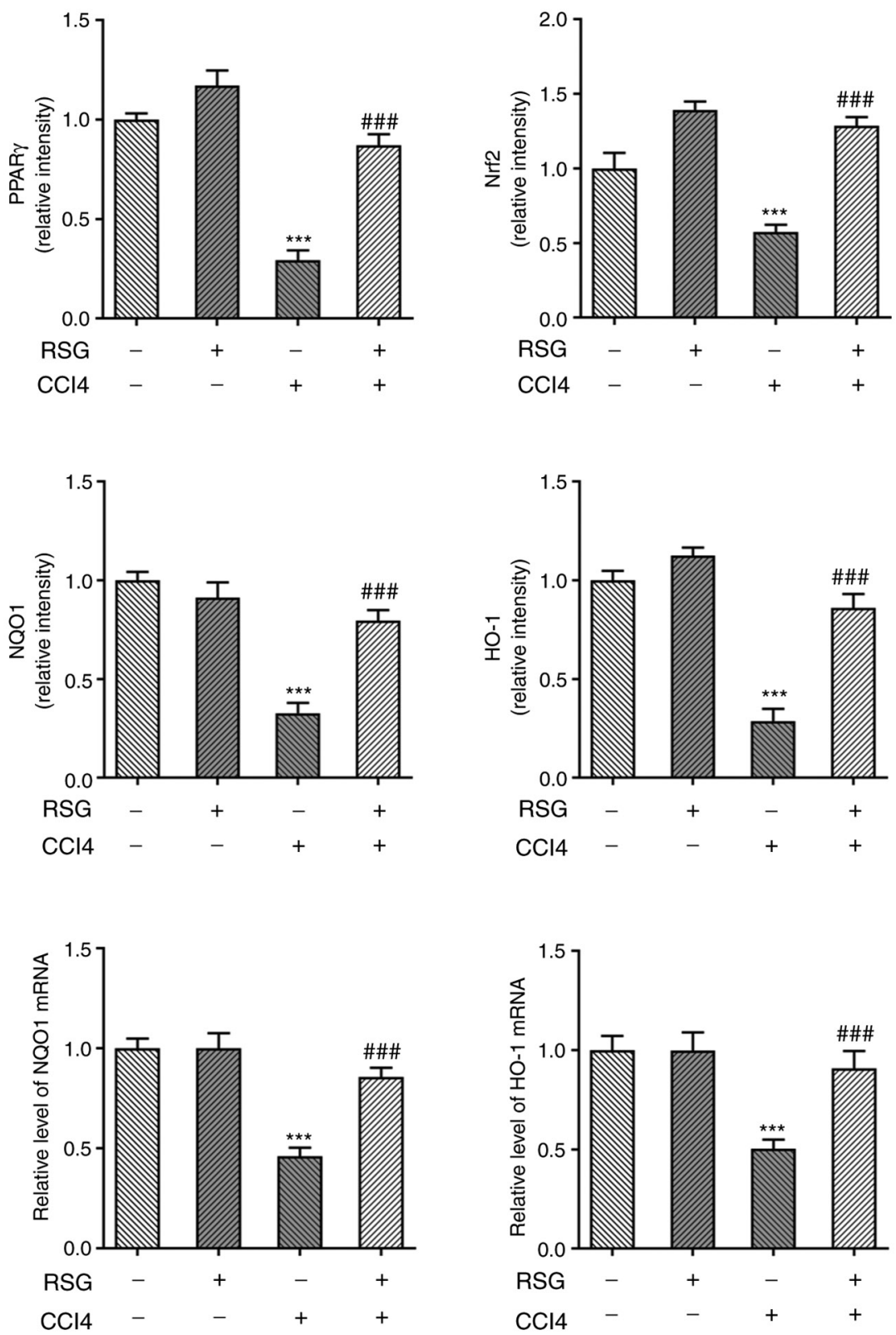

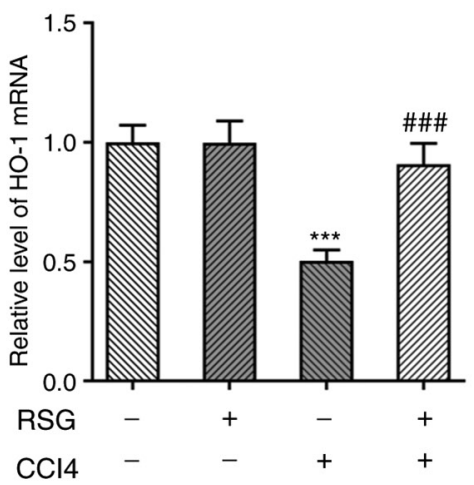

Figure 3. (A) PPAR $\gamma$ and Nrf2 signaling pathway-related protein expression levels were determined using western blot analysis. (B) mRNA expression levels of Nrf2, NQO1 and HO-1 were detected by reverse transcription-quantitative $\mathrm{PCR}$. ${ }^{* * *} \mathrm{P}<0.001$ vs. the control group; ${ }^{\# \# \# ~} \mathrm{P}<0.001$ vs. the $\mathrm{CCl} \mathrm{H}_{4}$ group. $\mathrm{RSG}$, rosiglitazone; $\mathrm{CCl}_{4}$, carbon tetrachloride; PPAR $\gamma$, peroxisome proliferator-activated receptor $\gamma ; \mathrm{Nrf} 2$, nuclear factor erythroid 2-related factor 2; NQO1, NAD(P) $\mathrm{H}$ quinone oxidoreductase 1 ; HO-1, heme oxygenase 1.

metal elements, reflects the organismal ability to scavenge oxygen free radicals (20). CAT, a scavenger enzyme removes hydrogen peroxide from the body (21). In addition, GSH, a small peptide composed of three amino acids, transforms free radicals to acidic substances, thereby accelerating the excretion of free radicals (22). MDA, the final product of lipid peroxidation, can destroy the structural and functional integrity of cell membranes, and the levels of MDA indirectly reflect the degree of oxidative stress (23). The results of the current study showed that RSG could reverse the $\mathrm{CCl}_{4}$-mediated decrease in the levels of SOD, CAT and GSH, and the increase in levels of MDA, NO and ROS, thus suggesting that RSG could exert an inhibitory effect on oxidative stress. In addition, the reduced levels of inflammatory factors (24) and apoptosis-related proteins (25) indicated that
RSG could likewise alleviate inflammation and apoptosis in the liver.

The liver is rich in mitochondria and is the dominant organ for the generation of ROS (26). Under normal conditions, ROS is produced in hepatocytes as a by-product of normal metabolism and detoxification. On the other hand, the antioxidant system is involved in the immediate degradation of the newly generated ROS in the liver (27). However, when the production of ROS is continuous, the excessive amounts of ROS can cause cell damage, eventually leading to the development of several hepatic diseases (28). Nrf2 is a key transcription factor that regulates the antioxidant stress response in cells and activates the endogenous antioxidant response (29). Under oxidative stress, Nrf2 dissociates from kelch-like ECH-associated protein 1 and dimerizes with several transcription factors to translocate to the nucleus. In 

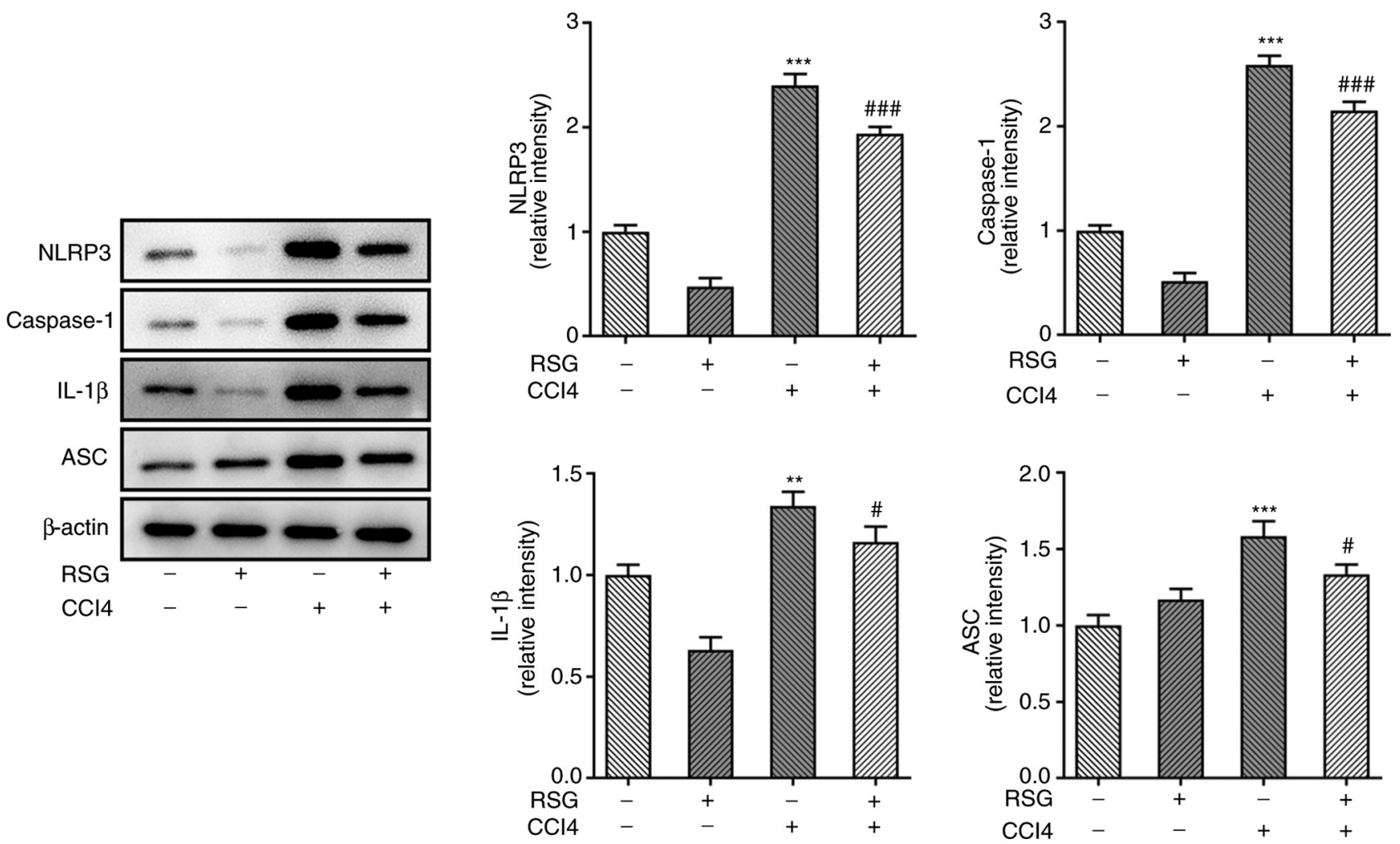

Figure 4. NLRP3 signaling pathway-related protein expression levels were determined using western blot analysis. ${ }^{* *} \mathrm{P}<0.01$ and ${ }^{* * *} \mathrm{P}<0.001$ vs. the control group; ${ }^{\#} \mathrm{P}<0.05$ and ${ }^{\# \# \# ~} \mathrm{P}<0.001$ vs. the $\mathrm{CCl}_{4}$ group. RSG, rosiglitazone; $\mathrm{CCl}_{4}$, carbon tetrachloride; ASC, apoptosis-associated speck-like protein; NLRP3, NOD-like receptor protein 3 .

the nucleus, Nrf2 regulates the expression of downstream factors, such as HO-1, NQO1 and other antioxidant enzymes (30). It has been reported that the Nrf2 signaling pathway is involved in the antioxidant defense system of the liver via regulating liver metabolism and detoxification, and promoting liver cell regeneration (31). More importantly, Nrf2 signaling is also involved in the pathogenesis of numerous hepatic diseases, such as drug-induced hepatic injury (32). A previous study demonstrated that excessive use of drugs may cause oxidative injury to the liver, thereby activating the Nrf2 pathway and promoting the rapid translocation of Nrf2 to the nucleus. In turn, activated Nrf2 could regulate the expression of its downstream target genes to exert its protective role in the liver (33). It has also been identified that the activation of NLRP3 signaling serves a significant role in acute hepatic injury (34). A previous study on mouse models indicated that NLRP3 may serve a key role in the modulation of hepatic inflammation and fibrosis (35). Additionally, NLRP3 may activate the proteolytic cleavage of pro-caspase-1 into activated caspase- 1 and is involved in the mature IL-1 $\beta$ - and IL-18-mediated inflammatory process. Mature IL-1 $\beta$ and IL-18 arise from the cytokine precursors pro-IL-1 $\beta$ and pro-IL-18, respectively (36). The results of the present study demonstrated that RSG could activate PPAR $\gamma$ and Nrf2, and inhibit the activation of the NLRP3 inflammasome.

In conclusion, the present study revealed that RSG could reduce the serum levels of ALT and AST, and indicated that it may inhibit inflammation, oxidative stress and hepatocyte apoptosis in a mouse model of hepatic injury. Notably, RSG could activate the Nrf2 signaling pathway and inhibit activation of the NLRP3 inflammasome, thus exerting a protective effect against acute hepatic injury. The current study may improve the understanding of the mechanism underlying the effect of RSG on hepatic injury, thus supporting the potential application of RSG in clinical practice. However, the present study is limited, as it only represents a preliminary mechanistic study; therefore, the mechanism of action of the downstream factors involved in the aforementioned pathways is worthy of further investigation.

\section{Acknowledgements}

Not applicable.

\section{Funding}

The present study was supported by the Eighth Affiliated Hospital of Xinjiang Medical University (grant no. 2020022).

\section{Availability of data and materials}

The datasets used and/or analyzed during the current study are available from the corresponding author on reasonable request.

\section{Authors' contributions}

MM and LM contributed to the conception of the study. MM wrote the manuscript. LM, YM, BXM and MM all performed the experiments. YM analyzed the data and BXM critically revised the manuscript. LM and MM confirm the authenticity of all raw data. All authors have read and approved the final version of the manuscript. 


\section{Ethics approval and consent to participate}

All animal experimental procedures were performed according to the ethical guidelines of The Eighth Affiliated Hospital of Xinjiang Medical University. All efforts were made to minimize animal suffering. This study was approved by the Institutional Animal Care and Use Committee of The Eighth Affiliated Hospital of Xinjiang Medical University (approval no. 2020-035).

\section{Patient consent for publication}

Not applicable.

\section{Competing interests}

The authors declare that they have no competing interests.

\section{References}

1. Coccolini F, Coimbra R, Ordonez C, Kluger Y, Vega F, Moore EE, Biffl W, Peitzman A, Horer T, Abu-Zidan FM, et al: Liver trauma: WSES 2020 guidelines. World J Emerg Surg 15: 24, 2020.

2. Zou Y, Xiong JB, Ma K, Wang AZ and Qian KJ: Rac2 deficiency attenuates $\mathrm{CCl}_{4}$-induced liver injury through suppressing inflammation and oxidative stress. Biomed Pharmacother 94: 140-149, 2017.

3. Zhang X, Kuang G, Wan J, Jiang R, Ma L, Gong X and Liu X Salidroside protects mice against CCl4-induced acute liver injury via down-regulating CYP2E1 expression and inhibiting NLRP3 inflammasome activation. Int Immunopharmacol 85: 106662, 2020

4. Cichoż-Lach $\mathrm{H}$ and Michalak A: Oxidative stress as a crucial factor in liver diseases. World J Gastroenterol 20: 8082-8091, 2014.

5. Li S, Tan HY, Wang N, Zhang ZJ, Lao L, Wong CW and Feng Y: The role of oxidative stress and antioxidants in liver diseases. Int J Mol Sci 16: 26087-26124, 2015.

6. Cong M, Zhao W, Liu T, Wang P, Fan X, Zhai Q, Bao X, Zhang D, You H, Kisseleva T, et al: Protective effect of human serum amyloid $\mathrm{P}$ on CCl4-induced acute liver injury in mice. Int J Mol Med 40: 454-464, 2017.

7. Tsai TH, Tam K, Chen SF, Liou JY, Tsai YC, Lee YM, Huang TY and Shyue SK: Deletion of caveolin-1 attenuates LPS/GalN-induced acute liver injury in mice. J Cell Mol Med 22: 5573-5582, 2018.

8. Stage TB, Christensen MH, Jørgensen NR, Beck-Nielsen H, Brøsen K, Gram J and Frost M: Effects of metformin, rosiglitazone and insulin on bone metabolism in patients with type 2 diabetes. Bone 112: 35-41, 2018.

9. Li J, Xue YM, Zhu B, Pan YH, Zhang Y, Wang C and Li Y: Rosiglitazone Elicits an Adiponectin-Mediated Insulin-Sensitizing Action at the Adipose Tissue-Liver Axis in Otsuka Long-Evans Tokushima Fatty Rats. J Diabetes Res 2018: $4627842,2018$.

10. Ahn KO, Lim SW, Yang HJ, Li C, Sugawara A, Ito S, Choi BS, Kim YS, Kim J and Yang CW: Induction of PPAR gamma mRNA and protein expression by rosiglitazone in chronic cyclosporine nephropathy in the rat. Yonsei Med J 48: 308-316, 2007.

11. Yu X, Shao XG, Sun H, Li YN, Yang J, Deng YC and Huang YG: Activation of cerebral peroxisome proliferator-activated receptors gamma exerts neuroprotection by inhibiting oxidative stress following pilocarpine-induced status epilepticus. Brain Res 1200: 146-158, 2008.

12. Gong P, Stewart D, Hu B, Li N, Cook J, Nel A and Alam J: Activation of the mouse heme oxygenase-1 gene by 15-deoxy-Delta(12,14)-prostaglandin $\mathrm{J}(2)$ is mediated by the stress response elements and transcription factor Nrf2. Antioxid Redox Signal 4: 249-257, 2002.

13. Wang X, Wang Z, Liu JZ, Hu JX, Chen HL, Li WL and Hai CX: Double antioxidant activities of rosiglitazone against high glucose-induced oxidative stress in hepatocyte. Toxicol In Vitro 25 839-847, 2011.

14. Ravan AP, Bahmani M, Ghasemi Basir HR, Salehi I and Oshaghi EA: Hepatoprotective effects of Vaccinium arctostaphylos against CCl4-induced acute liver injury in rats. J Basic Clin Physiol Pharmacol 28: 463-471, 2017.
15. National Research Council of the National Academies: Guide for the Care and Use of Laboratory Animals. 8th edition. The National Academies Press, Washington, DC, 2011.

16. Livak KJ and Schmittgen TD: Analysis of relative gene expression data using real-time quantitative PCR and the 2(-Delta Delta C(T)) Method. Methods 25: 402-408, 2001.

17. Farzanegi P,Dana A,EbrahimpoorZ,Asadi Mand Azarbayjani MA: Mechanisms of beneficial effects of exercise training on non-alcoholic fatty liver disease (NAFLD): Roles of oxidative stress and inflammation. Eur J Sport Sci 19: 994-1003, 2019.

18. Wei PC, Chang AN, Kao J, Du Z, Meyers RM, Alt FW and Schwer B: Long Neural Genes Harbor Recurrent DNA Break Clusters in Neural Stem/Progenitor Cells. Cell 164: 644-655, 2016.

19. Melchart D, Hager S, Albrecht S, Dai J, Weidenhammer W and Teschke R: Herbal Traditional Chinese Medicine and suspected liver injury: A prospective study. World J Hepatol 9: 1141-1157, 2017.

20. Dormandy TL: Free-radical pathology and medicine. A review. J R Coll Physicians Lond 23: 221-227, 1989.

21. Popović B, Velimirović M, Stojković T, Brajović G, De Luka SR, Milovanović I, Stefanović S, Nikolić D, Ristić-Djurović JL, Petronijević ND and Trbovich AM: The influence of ageing on the extrapineal melatonin synthetic pathway. Exp Gerontol 110: 151-157, 2018.

22. D'Agostino J, Zhang H, Kenaan $\mathrm{C}$ and Hollenberg PF: Mechanism-based inactivation of human cytochrome P450 2B6 by chlorpyrifos. Chem Res Toxicol 28: 1484-1495, 2015.

23. Cui X, Gong J, Han H, He L, Teng Y, Tetley T, Sinharay R, Chung KF, Islam T, Gilliland F, et al: Relationship between free and total malondialdehyde, a well-established marker of oxidative stress, in various types of human biospecimens. J Thorac Dis 10: 3088-3097, 2018.

24. Ohara M, Ohnishi S, Hosono H, Yamamoto K, Yuyama K, Nakamura H, Fu Q, Maehara O, Suda G and Sakamoto N: Extracellular vesicles from amnion-derived mesenchymal stem cells ameliorate hepatic inflammation and fibrosis in rats. Stem Cells Int 2018: 3212643, 2018.

25. Chen MF, Huang SJ, Huang CC, Liu PS, Lin KI, Liu CW, Hsieh WC, Shiu LY and Chen CH: Saikosaponin d induces cell death through caspase-3-dependent, caspase-3-independent and mitochondrial pathways in mammalian hepatic stellate cells. BMC Cancer 16: 532, 2016.

26. Mansouri A, Gattolliat $\mathrm{CH}$ and Asselah T: Mitochondrial dysfunction and signaling in chronic liver diseases. Gastroenterology 155: 629-647, 2018.

27. Okoye CN, MacDonald-Jay N and Kamunde C: Effects of bioenergetics, temperature and cadmium on liver mitochondria reactive oxygen species production and consumption. Aquat Toxicol 214: $105264,2019$.

28. Zhu R, Wang Y, Zhang L and Guo Q: Oxidative stress and liver disease. Hepatol Res 42: 741-749, 2012.

29. No JH, Kim YB and Song YS: Targeting nrf2 signaling to combat chemoresistance. J Cancer Prev 19: 111-117, 2014.

30. Bataille AM and Manautou JE: Nrf2: A potential target for new therapeutics in liver disease. Clin Pharmacol Ther 92: 340-348, 2012.

31. Dodson M, de la Vega MR, Cholanians AB, Schmidlin CJ, Chapman E and Zhang DD: Modulating NRF2 in disease: Timing is everything. Annu Rev Pharmacol Toxicol 59: 555-575, 2019.

32. Shin SM, Yang JH and Ki SH: Role of the Nrf2-ARE pathway in liver diseases. Oxid Med Cell Longev 2013: 763257, 2013.

33. Chan K, Han XD and Kan YW: An important function of Nrf2 in combating oxidative stress: Detoxification of acetaminophen. Proc Natl Acad Sci USA 98: 4611-4616, 2001.

34. Xu Y, Tang Y, Lu J, Zhang W, Zhu Y, Zhang S, Ma G, Jiang P and Zhang W: PINK1-mediated mitophagy protects against hepatic ischemia/reperfusion injury by restraining NLRP3 inflammasome activation. Free Radic Biol Med 160: 871-886, 2020.

35. Wree A, McGeough MD, Inzaugarat ME, Eguchi A, Schuster S, Johnson CD, Peña CA, Geisler LJ, Papouchado BG, Hoffman HM and Feldstein AE: NLRP3 inflammasome driven liver injury and fibrosis: Roles of IL-17 and TNF in mice. Hepatology 67: 736-749, 2018.

36. Strowig T, Henao-Mejia J, Elinav E and Flavell R: Inflammasomes in health and disease. Nature 481: 278-286, 2012.

This work is licensed under a Creative Commons Attribution-NonCommercial-NoDerivatives 4.0 International (CC BY-NC-ND 4.0) License. 